\title{
A mobile ion in a Fermi sea
}

\author{
Esben Rohan Christensen, ${ }^{1}$ Arturo Camacho-Guardian, ${ }^{2}$ and Georg M. Bruun ${ }^{1,3}$ \\ ${ }^{1}$ Center for Complex Quantum Systems, Department of Physics and Astronomy, \\ Aarhus University, Ny Munkegade 120, DK-8000 Aarhus C, Denmark. \\ ${ }^{2}$ T.C.M. Group, Cavendish Laboratory, University of Cambridge, \\ JJ Thomson Avenue, Cambridge, CB3 OHE, U.K. \\ ${ }^{3}$ Shenzhen Institute for Quantum Science and Engineering and Department of Physics, \\ Southern University of Science and Technology, Shenzhen 518055, China.
}

(Dated: November 1, 2021)

\begin{abstract}
The remarkable single particle control of individual ions combined with the versatility of ultracold atomic gases makes hybrid ion-atom system an exciting new platform for quantum simulation of few- and many-body quantum physics. Here, we study theoretically the properties of a mobile ion immersed in a quantum degenerate gas of fermionic atoms. Using an effective low-energy atom-ion interaction together with a well established approach that includes exactly two-body correlations, we calculate the full spectral response of the ion and demonstrate the existence of several quasiparticle branches, which are charged analogues of the Fermi polaron observed in neutral atomic gases. Due to the long-range nature of the atom-ion interaction, these ionic Fermi polarons have several properties distinct from their neutral counterparts such as the simultaneous presence of several stable states and smooth transitions from repulsive to attractive polarons with increasing interaction strength. Surprisingly, the residue of the ionic polaron is shown to increase with the Fermi density for fixed interaction strength, which is in marked contrast to the neutral polaron. The properties of the ionic polaron approach that of the neutral polaron only in the low density limit where the average interparticle spacing is larger than the characteristic length of the atom-ion interaction. We finally analyse the effects of the Fermi gas on the molecular ions, which are bound atom-dimer states.
\end{abstract}

\section{INTRODUCTION}

The ability to prepare and manipulate both single ions as well as neutral atomic gases has opened up opportunities to explore quantum systems consisting of ions embedded in a neutral gas environment [1]. Due to their hybrid ion-neutral nature, such systems represent a new powerful quantum simulation platform for investigating fundamental physics ranging from two-body collisional processes, molecule formation, to complex manybody states, and they may also have applications for future quantum information technologies. While the field of hybrid ultracold quantum ion-atom systems is still in its infancy, experiments have already explored ion-atom collisions and sympathetic cooling [2-5], threebody recombination, molecule formation, charge transfer reactions [6-10], ions in a Bose-Einstein condensate (BEC) [11], charge transport [12], and the use of ions for high resolution microscopy [13]. Theoretically, one has used a wide range of techniques to study the fewbody properties such as atom-ion scattering, three-body recombination and molecule formation, cold chemistry, as well as sympathetic cooling [14-19]. The many-body properties of these hybrid systems in the quantum degenerate regime have also been investigated theoretically with an important early exploring a static ion in a BoseEinstein condensate (BEC) using the Gross-Pitaevskii equation [20]. Later, calculations based on a weak coupling Fröhlich model [21], Monte-Carlo numerics [22] as well as a variational approach [23], predicted that immersing a mobile ion in a BEC can lead to the formation of quasiparticles, which are the charged analogue of the
Bose polaron observed for neutral impurity atoms in a BEC [24-27]. The atom-ion interaction is however much longer ranged than that between neutral atoms, and it is therefore presently unclear under which conditions this leads to strong three-body losses that may complicate the observation of such ionic Bose polarons $[6,10]$.

Immersing an ion in a single-component Fermi gas generally provides a more stable system since the Pauli exclusion principle suppresses three-body losses involving two identical fermions. As such, this provides a promising pathway to realise interesting quantum states such as charged quasiparticles in analogy with the observation of stable Fermi polarons formed by neutral impurity atoms in Fermi gases [28-32], where the interaction between the impurity and the environment is short-range [33]. Fermi polarons have recently also been observed in the context of exciton-polaritons [34], which are again well described theoretically using a short-range interaction [35-37]. The properties of Fermi polarons with long-range interactions remain on the other hand much less explored.

Motivated by this, we explore here the properties of a single mobile ion in a quantum degenerate gas of fermionic atoms. Using a low energy effective interaction combined with the simplest possible approach that includes exactly two-body atom-ion correlations known to give quantitative accurate results for neutral impurities in a Fermi gas, we calculate the full spectral response of the ion. We show that there are several quasiparticle branches corresponding to ionic Fermi polarons, which due to the long-range atom-ion interaction have properties quite different from the neutral Fermi polaron. This includes the simultaneous presence of several sta- 
ble polaron branches and the smooth transition from repulsive to attractive polarons with increasing interaction strength. Remarkably, the residue of the attractive polaron is demonstrated to increase with increasing Fermi density and remains non-zero in the so-called BEC limit in stark contrast to the residue of the neutral attractive polaron. Only when the density is so low that the average distance between the particles is larger than the characteristic length of the atom-ion interaction do the properties of the ionic polaron resemble those of the neutral polaron. We finally analyse the effects of the Fermi gas on the bound atom-ion states corresponding to molecular ions.

The manuscript is organised as follows. In Sec. II, we describe the hybrid atom-ion system, and in Sec. III we analyse the two-body atom-ion scattering and molecule formation using both a real space and a momentum space approach. The many-body problem is then explored in Sec. IV where the full spectral response of an ion in a Fermi gas is calculated. In Sec. V, we discuss the formation of molecular ions in the presence of the Fermi sea and we conclude and provide an outlook in Sec. VI.

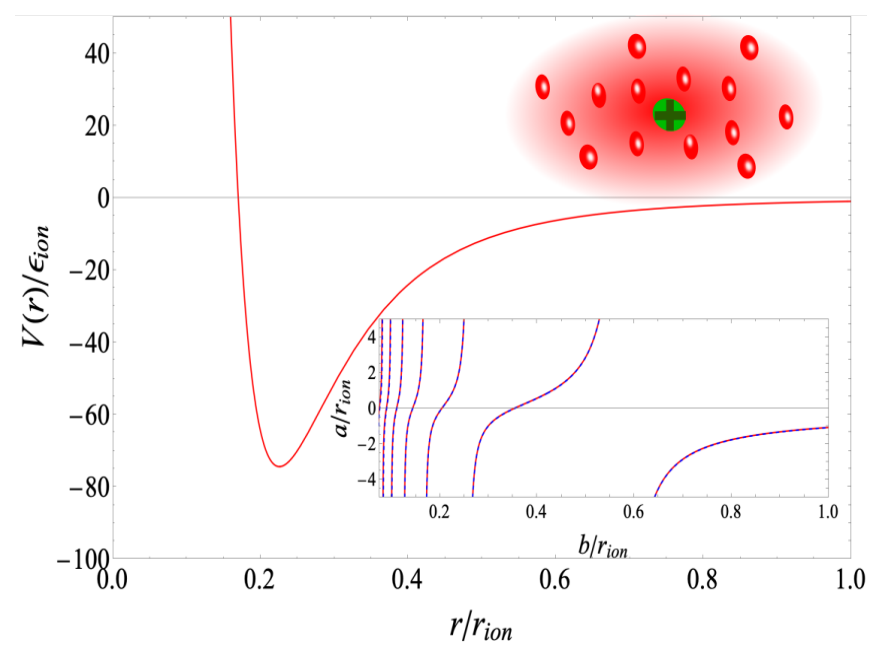

FIG. 1. The ion-atom interaction potential as a function of $r$. (Inset top) Illustration of a single ion in a single component Fermi gas. (Inset bottom) The atom-ion scattering length $a$ as a function of $b$ calculated by direct solution of the Schrödinger equation in real space (red line) and from the Lipmann-Schwinger equation (blue dashed line).

\section{SYSTEM}

We consider a single mobile ion of mass $m$ immersed in a gas of single-component fermionic atoms of mass $m_{F}$ at zero temperature. The Hamiltonian describing the system is

$$
\hat{H}=\sum_{\mathbf{k}}\left(\varepsilon_{\mathbf{k}} \hat{a}_{\mathbf{k}}^{\dagger} \hat{a}_{\mathbf{k}}+\varepsilon_{F \mathbf{k}} \hat{c}_{\mathbf{k}}^{\dagger} \hat{c}_{\mathbf{k}}\right)+\frac{1}{\mathcal{V}} \sum_{\mathbf{k}, \mathbf{k}^{\prime}, \mathbf{q}} V(\mathbf{q}) \hat{c}_{\mathbf{k}^{\prime}-\mathbf{q}}^{\dagger} \hat{c}_{\mathbf{k}^{\prime}} \hat{a}_{\mathbf{k}+\mathbf{q}}^{\dagger} \hat{a}_{\mathbf{k}}
$$

where $\hat{a}_{\mathbf{k}}^{\dagger}$ and $\hat{c}_{\mathbf{k}}^{\dagger}$ create an ion and a fermion with momentum $\mathbf{k}$ and energy $\varepsilon_{\mathbf{k}}=k^{2} / 2 m$ and $\varepsilon_{F \mathbf{k}}=k^{2} / 2 m_{F}$ respectively. The second term in Eq. (1) describes the ion-atom interaction and we can as usual neglect the short-range interaction between the spin-polarised fermions. Finally, $\mathcal{V}$ denotes the volume of the system and we take $\hbar=1$ throughout

\section{TWO-BODY PROPERTIES}

Before exploring the many-body problem, we start by discussing the relevant low energy atom-ion two-body physics. For a large separation $r$, the dominant source of the interaction comes from the electric field of the ion polarising the atom. This gives rise to an attractive interaction of the form $\sim-\alpha / r^{4}$ where $\alpha$ is proportional to the static electric polarisability of the atom and the charge of the ion. For short distances, the overlap between electron clouds of the atom and the ion gives rise to strong short-range repulsion. The full atom-ion interaction is quite complicated with many deeply bound states [1]. Since we focus on the low energy physics, it is sufficient to use an effective interaction of the form [38]

$$
V(r)=-\frac{\alpha}{\left(r^{2}+b^{2}\right)^{2}} \frac{r^{2}-c^{2}}{r^{2}+c^{2}} .
$$

Here, $b$ determines the depth of the potential, whereas $c$ determines distance below which the interaction is repulsive, see Fig. 1. The strong repulsion at short distances is accounted for by the fact that $V(0)=\alpha / b^{4}$ is much larger than any other relevant energy in the system. From the long-range behaviour $\sim-\alpha / r^{4}$ of the interaction, we can define the characteristic length $r_{\text {ion }}=\sqrt{2 m_{r} \alpha}$ and energy $\varepsilon_{\text {ion }}=1 / 2 m_{r} r_{\text {ion }}^{2}$ where $m_{r}=\left(m_{F} m\right) /\left(m+m_{F}\right)$ is the reduced mass [20].

The parameters $b$ and $c$ can be determined by requiring that the effective interaction Eq. (2) reproduces the correct low energy two-body physics relevant for the manybody problem at hand, i.e. the scattering length and energy of the highest bound state of a given atom-ion combination $[19,38]$. An ion with momentum $\mathbf{k}_{1}$ scattering on an atom with momentum $\mathbf{k}_{2}$ leading to the momentum transfer $\mathbf{q}$ is described by the scattering matrix, which obeys Lippmann-Schwinger equation

$$
\begin{gathered}
\mathcal{T}\left(\mathbf{k}_{1}, \mathbf{k}_{2}, \mathbf{q} ; E\right)=V(\mathbf{q})+\frac{1}{\mathcal{V}} \sum_{\mathbf{q}^{\prime}} V\left(\mathbf{q}^{\prime}\right) \times \\
\mathcal{G}_{2}\left(\mathbf{k}_{1}+\mathbf{q}^{\prime}, \mathbf{k}_{2}-\mathbf{q}^{\prime}, E\right) \mathcal{T}\left(\mathbf{k}_{1}+\mathbf{q}^{\prime}, \mathbf{k}_{2}-\mathbf{q}^{\prime}, \mathbf{q}-\mathbf{q}^{\prime} ; E\right) .
\end{gathered}
$$

Here, $E$ is the total energy and $\mathcal{G}_{2}^{-1}\left(\mathbf{k}_{1}, \mathbf{k}_{2}, E\right)=E-\varepsilon_{\mathbf{k}_{1}}-$ $\varepsilon_{F \mathbf{k}_{2}}$ describes the propagation of an ion and an atom 
with momenta $\mathbf{k}_{1}$ and $\mathbf{k}_{2}$ and total energy $E$. The $s$-wave scattering length can be obtained by expanding the zero center of mass scattering matrix in Legendre polynomials $\mathcal{T}(\mathbf{k},-\mathbf{k}, \mathbf{q} ; E)=\sum_{l=0}(l+1 / 2) \mathcal{T}_{l}(k, q ; E) P_{l}\left(\cos \theta_{\mathbf{k}, \mathbf{k}+\mathbf{q}}\right)$ giving

$$
a=\frac{2 \pi}{m_{r}} \mathcal{T}_{0}(0,0 ; 0) .
$$

We plot in Fig. 1 the scattering lengths obtained from this procedure as a function of $b$. For alkali atoms the interaction is repulsive for $r \lesssim 10 a_{0}$ while $r_{\text {ion }} \sim \mathcal{O}\left(10^{2}\right) \mathrm{nm}$ and we therefore take $c=0.0023 r_{\text {ion }}$ in this manuscript. We see that the scattering length exhibits several divergencies as the atom-ion interaction potential deepens with decreasing $b$, which are caused by the emergence of $s$-wave bound dimer states. The energies $\varepsilon_{M}$ of these molecular ions can be found as poles of the scattering matrix as a function of the energy.

We also show in Fig. 1 the scattering obtained from solving the Schrödinger equation $H \psi(r)=E \psi(r)$ in real space. For vanishing energy and zero angular momentum, the radial wave function takes the form $\psi(r)=$ $\chi(r) / r$, where $\chi(r)=c_{1} r+c_{2}$ for large $r$ and from this one can extract the scattering length as $a=-c_{2} / c_{1}$. The excellent agreement between the scattering length obtained from this real space method and from the LippmannSchwinger equation confirms the accuracy of our numerical calculations. We note that they are significantly more complicated than for the usual case of a short-range interaction, since one needs to retain the full momentum dependence of the atom-ion interaction.

\section{IONIC FERMI POLARON}

Now we turn our attention to the many-body problem of a mobile ion interacting with a single-component Fermi sea. The properties of an ion with momentum $\mathbf{p}$ are described by the Green's function

$$
\mathcal{G}^{-1}(\mathbf{p}, i \omega)=i \omega-\varepsilon_{\mathbf{p}}-\Sigma(\mathbf{p}, i \omega),
$$

where $\Sigma(\mathbf{p}, \omega)$ is the ion self-energy and $i \omega$ a Matsubara frequency. Inspired by its successful use for analysing neutral atomic Fermi gases, we use ladder approximation to calculate the self-energy. This is the simplest approach that includes the two-body atom-ion physics exactly in a many-body setting, and it gives

$$
\Sigma(\mathbf{p}, i \omega)=\sum_{\mathbf{q}, \omega_{q}} \mathcal{T}\left(\mathbf{p}, \mathbf{q}, \mathbf{0} ; i \omega+i \omega_{q}\right) G_{F}\left(\mathbf{q}, i \omega_{q}\right),
$$

where $G_{F}^{-1}\left(\mathbf{q}, i \omega_{q}\right)=i \omega_{q}-\varepsilon_{F \mathbf{q}}+\mu_{F}$ is the non-interacting Green's function of the atoms with $\mu_{F}$ their chemical potential. The scattering matrix $\mathcal{T}$ in Eq. (6) is obtained from the $s$-wave component of Eq. (3) using $\mathcal{G}_{2}\left(\mathbf{k}_{1}, \mathbf{k}_{2}, i \omega_{q}\right)=\left(1-f_{\mathbf{k}_{1}}\right) /\left(i \omega_{q}-\varepsilon_{\mathbf{k}_{1}}-\varepsilon_{F \mathbf{k}_{2}}+\mu_{F}\right)$, with $f_{\mathbf{k}}=\left[\exp \beta\left(\varepsilon_{F \mathbf{k}_{2}}-\mu_{F}\right)+1\right]^{-1}$ the Fermi distribution. This accounts for the Fermi blocking of the intermediate scattering states. Despite its simplicity, the ladder approximation turns out to be surprisingly accurate for neutral impurities in a Fermi gas [39]. This can be attributed to an almost perfect cancellation of higher order particlehole excitations due to Fermi statistics [40]. While such a cancellation may be less complete for the longer range atom-ion potential, we expect the ladder approximation to describe correctly the most important properties of mobile ions in a Fermi gas.

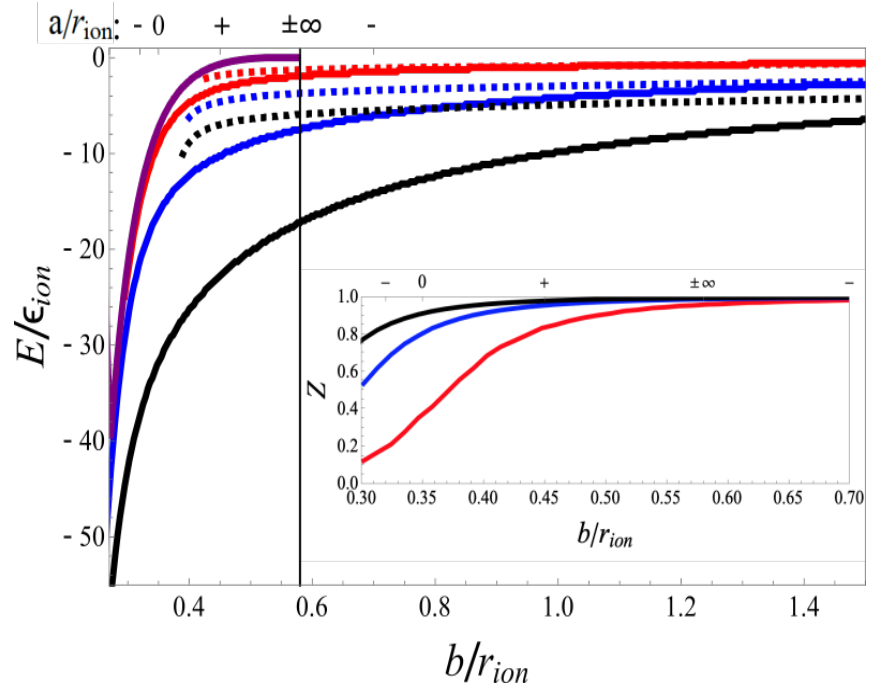

FIG. 2. Ionic polaron energy for the densities $n_{F} r_{\text {ion }}^{3}=0.1$ (red), $n_{F} r_{\text {ion }}^{3}=0.5$ (blue), and $n_{F} r_{\text {ion }}^{3}=1.0$ (black). The dashed lines give the energy of the neutral polaron for the same scattering length and densities, and the purple line gives the binding energy of the highest molecular ion. (Inset) Residue of the ionic polaron for the same densities as in the main panel using the same color scheme.

We start by studying the regime where there is at most one bound state in the atom-ion potential. In Fig. 2, we show the zero momentum quasiparticle energy $\varepsilon_{P}$ determined by the pole of the ion Green's function Eq. (5) taking $i \omega \rightarrow \omega+i 0_{+}$, i.e. solving $\varepsilon_{P}=\Sigma\left(\mathbf{0}, \varepsilon_{P}\right)$. The energy is plotted for three different densities $n_{F} r_{\text {ion }}^{3}=0.1,0.5$, and 1.0 where $n_{F}=\left(2 m_{F} \mu_{F}\right)^{3 / 2} / 6 \pi^{2}$, as a function of $b$ with the corresponding scattering length shown on top. This quasiparticle is the charged analogue of the Fermi polaron observed for neutral impurities in atomic gases [28-32], and we see that its energy decreases with increasing depth of the atom-ion potential and with the Fermi density $n_{F}$ as expected. The purple line gives the energy of the first bound state, which emerges for $b / r_{\text {ion }} \simeq 0.58$ indicated by a vertical black line. For comparison, we also show the energy obtained using the ladder approximation for a short-range interaction with the same scattering length a. Physically, this corresponds to the energy of the Fermi polaron for a neutral impurity where the interaction is short ranged and well described by the scattering length only [39]. We stop plotting the energy of this neutral polaron when its residue is below 0.05 . For low density 
$n_{F} r_{\text {ion }}^{3}=0.1$, the energy of the charged polaron is close to that of the neutral polaron. This reflects that the average distance between the particles is much larger than the characteristic length $r_{\text {ion }}$ of the atom-ion interaction so that it is well described by the scattering length only. The agreement however breaks down for strong interaction close to $b / r_{\text {ion }} \simeq 0.58$ where a bound state emerges and $1 / a=0$. Here, the atom-ion correlations are strong and depend on the range of the interaction. For larger density, the energy of the ionic polaron is considerably smaller than that of the neutral polaron except in the weakly interacting regime $n_{F}^{1 / 3} a \ll 1$, where it is given by the mean-field value $2 \pi a n_{F} / m_{r}$. This demonstrates that the scattering length approximation is insufficient when the average interparticle spacing is comparable to or smaller than the range the atom-ion interaction. It is also consistent with what is found for a mobile ion in a BEC [22, 23].

In the inset of Fig. 2, we show the quasiparticle residue obtained as $Z_{\mathbf{p}}^{-1}=1-\left.\partial_{\omega} \operatorname{Re} \Sigma(\mathbf{p}, \omega)\right|_{\varepsilon_{P}}$ for the same values of densities as in the main figure. Surprisingly, we see that the residue of the polaron increases with density. Intuitively, one would expect that the residue decreases since interaction effects should increase with density of the surrounding bath. Indeed, one finds that the residue of a neutral polaron interacting with a short-range potential decreases with increasing density. We also see from Fig. 2 that the polaron is a well-defined quasiparticle with a non-zero residue even for $a \rightarrow 0_{+}$, in stark contrast to the case of a neutral attractive polaron, where the residue vanishes in this so-called BEC limit [39]. These two effects can be understood from the fact that the energy $\varepsilon_{M}$ of the molecule present for $b / r_{\text {ion }}<0.58$ is higher than the value $-1 / 2 m_{r} a^{2}$ valid for a short range potential. In particular, it is finite for $a \rightarrow 0_{+}$as can be seen from Fig. 2 . It follows that for a large density $\epsilon_{F} / \varepsilon_{M}$ remains significant even when $a \rightarrow 0_{+}$leading to a non-zero residue of the ionic polaron. For a small density on the other hand, $\epsilon_{F} / \varepsilon_{M} \ll 1$ for $a \rightarrow 0_{+}$similar to a short range interaction leading to a small polaron residue. Equivalently, for a small density the polaron energy quickly approaches that of the molecule leading to a small residue, whereas the energy separation between the polaron and the molecule increases with the density leading to a larger residue. Fig. 2 therefore showcases that the long-range aspects of the ion-atom potential alter the properties of the ionic polaron compared to the neutral polaron.

To explore further the interplay between two- and many-body physics, we show in Fig. 3 the zero momentum ion spectral function defined as $A(\mathbf{p}, \omega)=$ $-2 \operatorname{ImG}(\mathbf{p}, \omega)$ as a function of $b$ both for low- and highdensity with $n_{F} r_{\text {ion }}^{3}=0.01$ and $n_{F} r_{\text {ion }}^{3}=1$. We see that each time the atom-ion interaction potential supports another bound state, a new polaron branch emerges. It follows that when there are $N$ molecular states, there are $N+1$ polaron branches in analogy with what has been predicted for a mobile ion in a BEC [20, 23]. However, the spectral weight of the low energy branches quickly decrease as the binding energy of the molecular states increase. This is especially apparent in the low density regime $n_{F} r_{\text {ion }}^{3}=0.01$ where the Fermi energy quickly becomes much smaller than the molecular binding energies corresponding to the BEC limit where the attractive polarons lose their spectral weight consistent with what we discussed above in connection with Fig. 2. The spectral weight is dominated by the highest energy polaron, which has an energy that agrees well with the mean-field prediction $2 \pi n_{F} a / m_{r}$ (red line) whenever $n_{F}|a|^{3} \ll 1$. This also means that it smoothly evolves from a repulsive $\varepsilon_{P}>0$ to an attractive $\varepsilon_{P}<0$ polaron with decreasing $b$. By comparing the two panels in Fig. 3, we see that the emergence of the new polaron branch is pushed to smaller values of $b$ for high density $n_{F} r_{\text {ion }}^{3}=1.0$ and that the lower polaron branch retains its spectral weight correspondingly longer. Also, the energy of the highest polaron quickly deviates from the mean-field prediction even when $n_{F}|a|^{3} \ll 1$, again illustrating the breakdown of the scattering length description when the interparticle distance becomes comparable to $r_{\text {ion }}$. Note that the effective interaction potential given by Eq. (2) can only be expected to reproduce the correct energy of the highest bound $s$-wave state of the real atom-ion interaction. Thus, while the general behaviour shown in Fig. 3 is robust, the spacing between the molecular resonances will depend on the specific atom-ion combination.

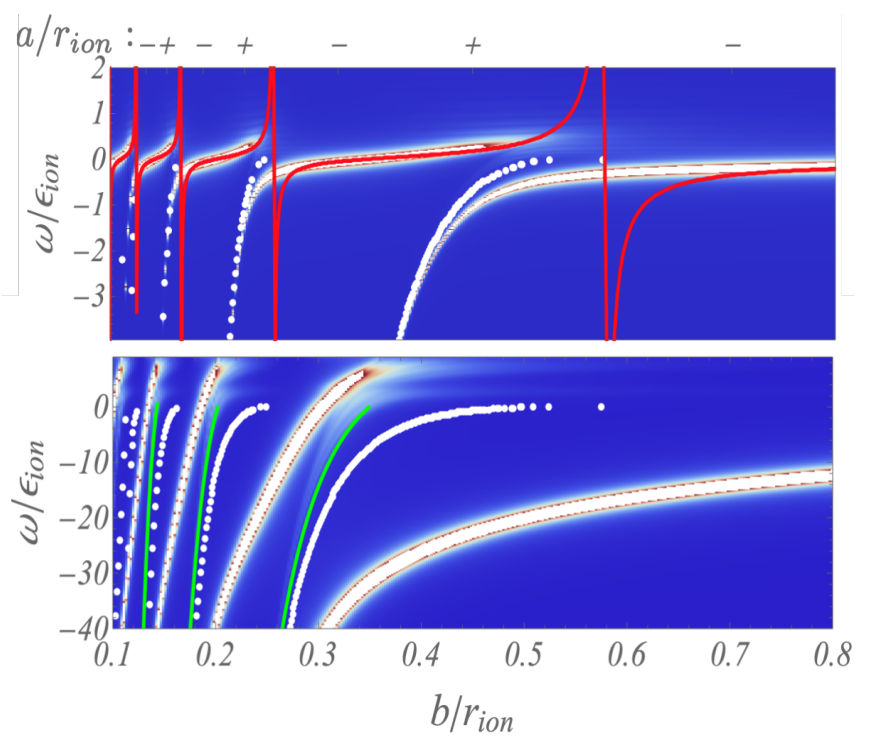

FIG. 3. Zero momentum spectral function of an ion. (top) Low-density regime corresponding to $n_{F} r_{\text {ion }}^{3}=0.01$, and highdensity regime $n_{F} r_{\text {ion }}^{3}=1.0$ in (bottom). The white dots depict the binding energy $\epsilon_{B}^{(0)}$, the red line corresponds to the mean-field energy. The molecule energy in presence of the Fermi sea is shown by the green lines (bottom).

We plot in Fig. 4 the energy of the zero momentum polaron as a function of the density for $b / r_{\text {ion }}=2$ and $b / r_{\text {ion }}=0.3237$ (the highest polaron branch), which both correspond to the scattering length $a / r_{\text {ion }} \simeq-0.42$. For 
low densities, the two polaron energies are accurately described by the mean-field expression $\varepsilon_{P}=2 \pi a n_{F} / m_{r}$ (black dashed line). The agreement breaks down with increasing density for the polaron at $b / r_{\text {ion }}=0.3237$. Such different properties of the two highest polaron branches sharing the same scattering length can be explained as follows. There is only one polaron branch at $b / r_{\text {ion }}=2$ and no molecular state, whereas the highest polaron for $b / r_{\text {ion }}=0.3237$ is above both a molecular state and a lower polaron branch. As the density increases, this upper polaron branch is therefore pushed away from the molecular state and the lower polaron branch. Since these effects are not included in mean-field theory, it has a smaller regime of validity for the highest polaron branch at $b / r_{\text {ion }}=0.3237$ compared to that at $b / r_{\text {ion }}=2$.

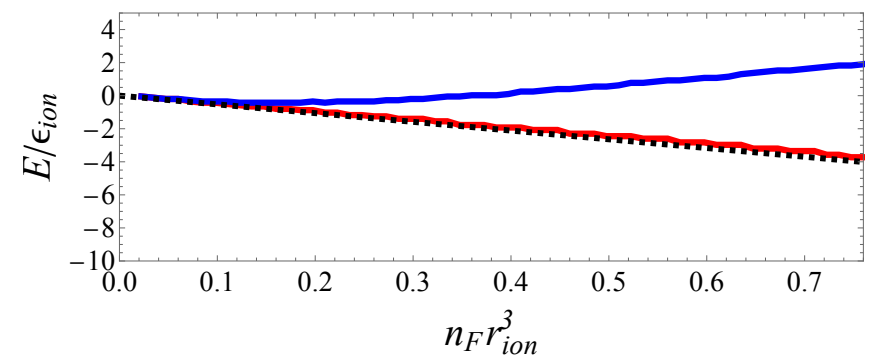

FIG. 4. The energy of the highest polaron branch as a function of density for $b / r_{\text {ion }}=2.0$ (red) and $b / r_{\text {ion }}=0.32$ (blue), which both correspond to the scattering length $a / r_{\text {ion }}=-0.42$. The mean-field prediction is illustrated by the dashed black line.

\section{IONIC MOLECULE}

We finally turn our attention to medium effects on the molecular ions. Inspired by a similar approach for neutral impurities in a Fermi gas [39, 41-43], we can write down the variational ansatz

$$
\left|\psi_{M}\right\rangle=\left(\sum_{\mathbf{k}} \phi_{\mathbf{k}} \hat{c}_{\mathbf{k}}^{\dagger} \hat{a}_{-\mathbf{k}}^{\dagger}+\sum_{\mathbf{k k}^{\prime} \mathbf{q}} \kappa_{\mathbf{k k}^{\prime} \mathbf{q}} \hat{c}_{\mathbf{k}}^{\dagger} \hat{c}_{\mathbf{k}^{\prime}}^{\dagger} \hat{c}_{\mathbf{q}} \hat{a}_{\mathbf{q}-\mathbf{k}-\mathbf{k}^{\prime}}^{\dagger}\right)|\mathrm{FS}\rangle
$$

to describe these dimer states in the presence of the Fermi sea $|\mathrm{FS}\rangle$ focusing on zero total momentum. Here, $\phi_{\mathbf{k}}$ and $\kappa_{\mathbf{k k}^{\prime} \mathbf{q}}$ are variational parameters, which are determined by minimising the energy $\left\langle\psi_{M}|H| \psi_{M}\right\rangle$. The details of this calculation and the resulting variational equations for $\phi_{\mathbf{k}}$ and $\kappa_{\mathbf{k k}^{\prime} \mathbf{q}}$ are presented in the Appendix. For simplicity, we present results for the molecule energy including only the first term in Eq. (7) taking $\kappa_{\mathbf{k k}^{\prime} \mathbf{q}}=0$.

The energy of the molecular ion obtained from Eq. (7) is plotted in the Fig. 3 as solid green lines. We see that the presence of the Fermi sea increases the molecule energy as compared to the vacuum case, which can easily be understood in terms of Fermi blocking of the available states for the molecular wave function. This effect therefore increases with the density, and we have not plotted the energy predicted by Eq. (7) for the low density case $n_{F} r_{\text {ion }}^{3}$ as it is indistinguishable from the molecule energy in a vacuum.

\section{CONCLUSIONS AND OUTLOOK}

We studied a single mobile ion in a quantum degenerate single-component Fermi gas. By calculating the full spectral response of the ion, we showed that the long-range atom-ion interaction gives rise to the presence of several ionic Fermi polaron branches, which have quite different properties as compared to neutral Fermi polarons. The ionic and neutral polarons were demonstrated to have similar properties only in the dilute limit where the average interparticle spacing is large compared to the characteristic length of the atomic interaction. We finally showed the effects of the Fermi gas on the atomion bound states states, i.e. the molecular ions.

Our results illustrate the rich physics that can be realised in hybrid ion-atom gases, and motivate future work. In particular, it would be highly relevant to calculate the three-body recombination rate for molecule formation for an ion in a degenerate Bose and Fermi gas to explore the lifetime of these systems [44-46]. This could be compared to neutral Bose and Fermi gases, where the three-body rate has been shown to depend on the scattering length as a simple power law [47-52]. Also, while the ladder approximation has been shown to be quantitatively reliable for a neutral impurity in a Fermi gas, an interesting question concerns its accuracy for the case of an ion where the long-range nature of the atom-ion interaction might give rise to significant corrections. Likewise, a remaining problem concerns the inclusion of more terms in the ansatz for the molecular ion, which might lead to a crossing of its energy with the polaron energy, as is the case for neutral impurities [41-43]. Finally, it would be interesting to calculate the induced interaction between two ions mediated by density oscillations in the surrounding medium, which in the case of neutral impurities can be quite strong leading to the formation of bound states [53, 54]. An accurate way to measure such an induced interaction could be to measure its effect on the phonon modes of ions confined in a radiofrequency or dipole trap $[4,5]$.

Acknowledgments.- This work has been supported by the Danish National Research Foundation through the Center of Excellence "CCQ" (Grant agreement no.: DNRF156), the Independent Research Fund DenmarkNatural Sciences via Grant No. DFF -8021-00233B, and the U.S. Army CCDC Atlantic Basic and Applied Research via Grant No. W911NF-19-1-0403. 
[1] Michał Tomza, Krzysztof Jachymski, Rene Gerritsma, Antonio Negretti, Tommaso Calarco, Zbigniew Idziaszek, and Paul S. Julienne, "Cold hybrid ion-atom systems," Rev. Mod. Phys. 91, 035001 (2019).

[2] Andrew T. Grier, Marko Cetina, Fedja Oručević, and Vladan Vuletić, "Observation of cold collisions between trapped ions and trapped atoms," Phys. Rev. Lett. 102, 223201 (2009).

[3] Christoph Zipkes, Stefan Palzer, Carlo Sias, and Michael Köhl, "A trapped single ion inside a bose-einstein condensate," Nature 464, 388-391 (2010).

[4] T. Feldker, H. Fürst, H. Hirzler, N. V. Ewald, M. Mazzanti, D. Wiater, M. Tomza, and R. Gerritsma, "Buffer gas cooling of a trapped ion to the quantum regime," Nature Physics 16, 413-416 (2020).

[5] J. Schmidt, P. Weckesser, F. Thielemann, T. Schaetz, and L. Karpa, "Optical traps for sympathetic cooling of ions with ultracold neutral atoms," Phys. Rev. Lett. 124, 053402 (2020).

[6] Arne Härter, Artjom Krükow, Andreas Brunner, Wolfgang Schnitzler, Stefan Schmid, and Johannes Hecker Denschlag, "Single ion as a three-body reaction center in an ultracold atomic gas," Phys. Rev. Lett. 109, 123201 (2012).

[7] Lothar Ratschbacher, Christoph Zipkes, Carlo Sias, and Michael Köhl, "Controlling chemical reactions of a single particle," Nature Physics 8, 649-652 (2012).

[8] Tomas Sikorsky, Ziv Meir, Ruti Ben-shlomi, Nitzan Akerman, and Roee Ozeri, "Spin-controlled atom-ion chemistry," Nature Communications 9, 920 (2018).

[9] T. Dieterle, M. Berngruber, C. Hölzl, R. Löw, K. Jachymski, T. Pfau, and F. Meinert, "Inelastic collision dynamics of a single cold ion immersed in a boseeinstein condensate," Phys. Rev. A 102, 041301 (2020).

[10] Amir Mohammadi, Artjom Krükow, Amir Mahdian, Markus Deiß, Jesús Pérez-Ríos, Humberto da Silva, Maurice Raoult, Olivier Dulieu, and Johannes Hecker Denschlag, "Life and death of a cold barb" molecule inside an ultracold cloud of rb atoms," Phys. Rev. Research 3, 013196 (2021).

[11] K. S. Kleinbach, F. Engel, T. Dieterle, R. Löw, T. Pfau, and F. Meinert, "Ionic impurity in a bose-einstein condensate at submicrokelvin temperatures," Phys. Rev. Lett. 120, 193401 (2018).

[12] T. Dieterle, M. Berngruber, C. Hölzl, R. Löw, K. Jachymski, T. Pfau, and F. Meinert, "Transport of a single cold ion immersed in a bose-einstein condensate," Phys. Rev. Lett. 126, 033401 (2021).

[13] C. Veit, N. Zuber, O. A. Herrera-Sancho, V. S. V. Anasuri, T. Schmid, F. Meinert, R. Löw, and T. Pfau, "Pulsed ion microscope to probe quantum gases," Phys. Rev. X 11, 011036 (2021).

[14] R. Côté, V. Kharchenko, and M. D. Lukin, "Mesoscopic molecular ions in bose-einstein condensates," Phys. Rev. Lett. 89, 093001 (2002).

[15] Bo Gao, "Universal properties in ultracold ion-atom interactions," Phys. Rev. Lett. 104, 213201 (2010).

[16] Artjom Krükow, Amir Mohammadi, Arne Härter, Johannes Hecker Denschlag, Jesús Pérez-Ríos, and Chris H. Greene, "Energy scaling of cold atom-atom-ion three-body recombination," Phys. Rev. Lett. 116, 193201
(2016).

[17] J. M. Schurer, A. Negretti, and P. Schmelcher, "Unraveling the structure of ultracold mesoscopic collinear molecular ions," Phys. Rev. Lett. 119, 063001 (2017).

[18] J. Pérez-Ríos, "Cold chemistry: a few-body perspective on impurity physics of a single ion in an ultracold bath," Molecular Physics 119, e1881637 (2021), https://doi.org/10.1080/00268976.2021.1881637.

[19] Lorenzo Oghittu, Melf Johannsen, Rene Gerritsma, and Antonio Negretti, "Dynamics of a trapped ion in a quantum gas: effects of particle statistics," (2021), arXiv:2109.03143 [cond-mat.quant-gas].

[20] P. Massignan, C. J. Pethick, and H. Smith, "Static properties of positive ions in atomic bose-einstein condensates," Phys. Rev. A 71, 023606 (2005).

[21] W. Casteels, J. Tempere, and J. T. Devreese, "Polaronic properties of an ion in a bose-einstein condensate in the strong-coupling limit," Journal of Low Temperature Physics 162, 266-273 (2011).

[22] Grigory E. Astrakharchik, Luis A. Peña Ardila, Richard Schmidt, Krzysztof Jachymski, and Antonio Negretti, "Ionic polaron in a bose-einstein condensate," Communications Physics 4, 94 (2021).

[23] Esben Rohan Christensen, Arturo Camacho-Guardian, and Georg M. Bruun, "Charged polarons and molecules in a bose-einstein condensate," Phys. Rev. Lett. 126, 243001 (2021).

[24] Nils B. Jørgensen, Lars Wacker, Kristoffer T. Skalmstang, Meera M. Parish, Jesper Levinsen, Rasmus S. Christensen, Georg M. Bruun, and Jan J. Arlt, "Observation of attractive and repulsive polarons in a boseeinstein condensate," Phys. Rev. Lett. 117, 055302 (2016).

[25] Ming-Guang Hu, Michael J. Van de Graaff, Dhruv Kedar, John P. Corson, Eric A. Cornell, and Deborah S. Jin, "Bose polarons in the strongly interacting regime," Phys. Rev. Lett. 117, 055301 (2016).

[26] L. A. Peña Ardila, N. B. Jørgensen, T. Pohl, S. Giorgini, G. M. Bruun, and J. J. Arlt, "Analyzing a bose polaron across resonant interactions," Phys. Rev. A 99, 063607 (2019).

[27] Zoe Z. Yan, Yiqi Ni, Carsten Robens, and Martin W. Zwierlein, "Bose polarons near quantum criticality," Science 368, 190-194 (2020), https://science.sciencemag.org/content/368/6487/190.full.pdf.

[28] André Schirotzek, Cheng-Hsun Wu, Ariel Sommer, and Martin W. Zwierlein, "Observation of fermi polarons in a tunable fermi liquid of ultracold atoms," Phys. Rev. Lett. 102, 230402 (2009).

[29] Christoph Kohstall, Mattheo Zaccanti, Matthias Jag, Andreas Trenkwalder, Pietro Massignan, Georg M Bruun, Florian Schreck, and Rudolf Grimm, "Metastability and coherence of repulsive polarons in a strongly interacting Fermi mixture," Nature 485, 615-618 (2012).

[30] Marco Koschorreck, Daniel Pertot, Enrico Vogt, Bernd Fröhlich, Michael Feld, and Michael Köhl, "Attractive and repulsive Fermi polarons in two dimensions," Nature 485, 619-622 (2012).

[31] F. Scazza, G. Valtolina, P. Massignan, A. Recati, A. Amico, A. Burchianti, C. Fort, M. Inguscio, M. Zaccanti, and G. Roati, "Repulsive fermi polarons in a resonant 
mixture of ultracold ${ }^{6} \mathrm{Li}$ atoms," Phys. Rev. Lett. 118, 083602 (2017).

[32] Isabella Fritsche, Cosetta Baroni, Erich Dobler, Emil Kirilov, Bo Huang, Rudolf Grimm, Georg M. Bruun, and Pietro Massignan, "Stability and breakdown of fermi polarons in a strongly interacting fermi-bose mixture," Phys. Rev. A 103, 053314 (2021).

[33] C. J. Pethick and H Smith, Bose-Einstein Condensation in Dilute Gases, 2nd ed. (Cambridge University Press, 2008).

[34] Meinrad Sidler, Patrick Back, Ovidiu Cotlet, Ajit Srivastava, Thomas Fink, Martin Kroner, Eugene Demler, and Atac Imamoglu, "Fermi polaron-polaritons in charge-tunable atomically thin semiconductors," Nature Physics 13, 255 EP - (2016).

[35] Dmitry K. Efimkin and Allan H. MacDonald, "Manybody theory of trion absorption features in twodimensional semiconductors," Phys. Rev. B 95, 035417 (2017).

[36] Li Bing Tan, Ovidiu Cotlet, Andrea Bergschneider, Richard Schmidt, Patrick Back, Yuya Shimazaki, Martin Kroner, and Atac Imamoglu, "Optical Amplification by Stimulated Cooling of Polaron-Polaritons," arXiv e-prints , arXiv:1903.05640 (2019), arXiv:1903.05640 [cond-mat.mes-hall].

[37] Miguel A. Bastarrachea-Magnani, Arturo CamachoGuardian, and Georg M. Bruun, "Attractive and repulsive exciton-polariton interactions mediated by an electron gas," Phys. Rev. Lett. 126, 127405 (2021).

[38] Michał Krych and Zbigniew Idziaszek, "Description of ion motion in a paul trap immersed in a cold atomic gas," Phys. Rev. A 91, 023430 (2015).

[39] Pietro Massignan, Matteo Zaccanti, and Georg M Bruun, "Polarons, dressed molecules and itinerant ferromagnetism in ultracold fermi gases," Rep. Progr. Phys. 77, 034401 (2014).

[40] R. Combescot and S. Giraud, "Normal state of highly polarized fermi gases: Full many-body treatment," Phys. Rev. Lett. 101, 050404 (2008).

[41] Christophe Mora and Frédéric Chevy, "Ground state of a tightly bound composite dimer immersed in a fermi sea," Phys. Rev. A 80, 033607 (2009).

[42] M. Punk, P. T. Dumitrescu, and W. Zwerger, "Polaronto-molecule transition in a strongly imbalanced fermi gas," Phys. Rev. A 80, 053605 (2009).

[43] R. Combescot, S. Giraud, and X. Leyronas, "Analytical theory of the dressed bound state in highly polarized Fermi gases," EPL 88, 60007 (2009).

[44] Jesús Pérez-Ríos and Chris H. Greene, "Communication: Classical threshold law for ion-neutral-neutral threebody recombination," The Journal of Chemical Physics 143, 041105 (2015), https://doi.org/10.1063/1.4927702.

[45] Bin-Bin Wang, Yong-Chang Han, Wei Gao, and Shu-Lin Cong, "Cold atom-atom-ion three-body recombination of 4he-4he-x- (x = h or d)," Phys. Chem. Chem. Phys. 19, 22926-22933 (2017).

[46] Bin-Bin Wang, Su-Hua Jing, and Ti-Xian Zeng, "Cold atom-atom-anion three-body recombination of 4 he4hexli(x =6 or 7) systems," The Journal of Chemical Physics 150, 094301 (2019), https://doi.org/10.1063/1.5087522.

[47] P. O. Fedichev, M. W. Reynolds, and G. V. Shlyapnikov, "Three-body recombination of ultracold atoms to a weakly bound $s$ level," Phys. Rev. Lett. 77, 2921-2924 (1996).
[48] Esben Nielsen and J. H. Macek, "Low-energy recombination of identical bosons by three-body collisions," Phys. Rev. Lett. 83, 1566-1569 (1999).

[49] B. D. Esry, Chris H. Greene, and James P. Burke, "Recombination of three atoms in the ultracold limit," Phys. Rev. Lett. 83, 1751-1754 (1999).

[50] P. F. Bedaque, Eric Braaten, and H.-W. Hammer, "Three-body recombination in bose gases with large scattering length," Phys. Rev. Lett. 85, 908-911 (2000).

[51] Tino Weber, Jens Herbig, Michael Mark, HannsChristoph Nägerl, and Rudolf Grimm, "Three-body recombination at large scattering lengths in an ultracold atomic gas," Phys. Rev. Lett. 91, 123201 (2003).

[52] D. S. Petrov, "Three-body problem in fermi gases with short-range interparticle interaction," Phys. Rev. A 67, 010703 (2003).

[53] A. Camacho-Guardian and Georg M. Bruun, "Landau effective interaction between quasiparticles in a boseeinstein condensate," Phys. Rev. X 8, 031042 (2018).

[54] A. Camacho-Guardian, L. A. Peña Ardila, T. Pohl, and G. M. Bruun, "Bipolarons in a bose-einstein condensate," Phys. Rev. Lett. 121, 013401 (2018). 


\section{APPENDIX}

\section{Appendix A: Dressed dimer equations}

We derive minimization equations for the dressed dimer with full ion potential. The Hamiltonian is still

$$
\hat{H}=\sum_{\mathbf{k}}\left(\varepsilon_{\mathbf{k}}^{I} \hat{f}_{\mathbf{k}}^{\dagger} \hat{f}_{\mathbf{k}}+\varepsilon_{F \mathbf{k}} \hat{c}_{\mathbf{k}}^{\dagger} \hat{c}_{\mathbf{k}}\right)+\sum_{\mathbf{k}, \mathbf{k}^{\prime}, \mathbf{q}} V(\mathbf{q}) \hat{c}_{\mathbf{k}^{\prime}-\mathbf{q}}^{\dagger} \hat{c}_{\mathbf{k}^{\prime}} \hat{f}_{\mathbf{k}+\mathbf{q}}^{\dagger} \hat{f}_{\mathbf{k}}
$$

while the ansatz can be conveniently written as

$$
\left|\psi^{D}\right\rangle=(\underbrace{\sum_{\mathbf{k}}^{\prime} \phi_{\mathbf{k}} \hat{c}_{\mathbf{k}}^{\dagger} \hat{f}_{-\mathbf{k}}^{\dagger}}_{\psi_{0}^{D}}+\underbrace{\sum_{\mathbf{k} \mathbf{k}^{\prime} \mathbf{q}}^{\prime} \kappa_{\mathbf{k} \mathbf{k}^{\prime} \mathbf{q}} \hat{c}_{\mathbf{k}}^{\dagger} \hat{c}_{\mathbf{k}^{\prime}}^{\dagger} \hat{c}_{\mathbf{q}} \hat{f}_{\mathbf{q}-\mathbf{k}-\mathbf{k}^{\prime}}^{\dagger}}_{\psi_{1}^{D}})\left|F S^{N}\right\rangle .
$$

We calculate expectation values and show each term explicitly for the interested reader:

$$
\begin{aligned}
& \left\langle\psi_{0}^{D}\left|H_{0}\right| \psi_{0}^{D}\right\rangle=\sum_{\mathbf{k}}^{\prime} \phi_{\mathbf{k}} \phi_{\mathbf{k}}^{*}\left(F \epsilon_{\mathbf{k}}+\epsilon_{\mathbf{k}}^{I}-\epsilon_{F}\right) \\
& \left\langle\psi_{1}^{D}\left|H_{0}\right| \psi_{1}^{D}\right\rangle=\sum_{\mathbf{k} \mathbf{k}^{\prime} \mathbf{q}}^{\prime}\left(\kappa_{\mathbf{k k}^{\prime} \mathbf{q}} \kappa_{\mathbf{k k}^{\prime} \mathbf{q}}^{*}-\kappa_{\mathbf{k} \mathbf{k}^{\prime} \mathbf{q}} \kappa_{\mathbf{k}^{\prime} \mathbf{k q}}^{*}\right)\left(\epsilon_{\mathbf{q}-\mathbf{k}-\mathbf{k}^{\prime}}^{I}+\epsilon_{F \mathbf{k}}+\epsilon_{F \mathbf{k}^{\prime}}-\epsilon_{F \mathbf{q}}+\sum_{\mathbf{q}^{\prime}}^{\prime} \epsilon_{F \mathbf{q}^{\prime}}-\epsilon_{F}\right) \\
& \left\langle\psi_{0}^{D}\left|H_{I}\right| \psi_{0}^{D}\right\rangle=\sum_{\mathbf{k k}^{\prime}}^{\prime} \phi_{\mathbf{k}} \phi_{\mathbf{k}^{\prime}}^{*} V\left(\mathbf{k}^{\prime}-\mathbf{k}\right)+V(\mathbf{0}) n_{F} \sum_{\mathbf{k}}^{\prime} \phi_{\mathbf{k}} \phi_{\mathbf{k}}^{*} \\
& \left\langle\psi_{1}^{D}\left|H_{I}\right| \psi_{1}^{D}\right\rangle=\sum_{\mathbf{k k}^{\prime} \mathbf{q} \mathbf{q}^{\prime}}^{\prime}\left(\kappa_{\mathbf{k k}^{\prime} \mathbf{q}} \kappa_{\mathbf{k}^{\prime} \mathbf{q}^{\prime}}^{*}-\kappa_{\mathbf{k k}^{\prime} \mathbf{q}} \kappa_{\mathbf{k}^{\prime} \mathbf{k} \mathbf{q}^{\prime}}^{*}\right) V\left(\mathbf{q}-\mathbf{q}^{\prime}\right)+\sum_{\mathbf{k k}_{\mathbf{1}} \mathbf{k}_{\mathbf{2}} \mathbf{q}}^{\prime}\left(\kappa_{\mathbf{k}_{\mathbf{1}} \mathbf{k q}} \kappa_{\mathbf{k}_{\mathbf{2}} \mathbf{k q}}^{*}-\kappa_{\mathbf{k}_{\mathbf{1}} \mathbf{k} \mathbf{q}} \kappa_{\mathbf{k}_{\mathbf{2}} \mathbf{q}}^{*}\right) V\left(\mathbf{k}_{\mathbf{2}}-\mathbf{k}_{\mathbf{1}}\right) \\
& +\sum_{\mathbf{k k}_{1} \mathbf{k}_{\mathbf{2}} \mathbf{q}}^{\prime}\left(\kappa_{\mathbf{k k}_{\mathbf{1}} \mathbf{q}} \kappa_{\mathbf{k k}_{\mathbf{2}} \mathbf{q}}^{*}-\kappa_{\mathbf{k k}_{\mathbf{1}} \mathbf{q}} \kappa_{\mathbf{k}_{\mathbf{2}} \mathbf{k q}}^{*}\right) V\left(\mathbf{k}_{\mathbf{2}}-\mathbf{k}_{\mathbf{1}}\right)+V(\mathbf{0}) n_{F} \sum_{\mathbf{k k}^{\prime} \mathbf{q}}^{\prime}\left(\kappa_{\mathbf{k k}^{\prime} \mathbf{q}} \kappa_{\mathbf{k}^{\prime} \mathbf{q}}^{*}-\kappa_{\mathbf{k k}^{\prime} \mathbf{q}} \kappa_{\mathbf{k}^{\prime} \mathbf{k q}}^{*}\right) \\
& \left\langle\psi_{1}^{D}\left|H_{I}\right| \psi_{0}^{D}\right\rangle=\sum_{\mathbf{k k}^{\prime} \mathbf{q}}^{\prime} \kappa_{\mathbf{k k}^{\prime} \mathbf{q}}^{*}\left(\phi_{\mathbf{k}^{\prime}} V(\mathbf{q}-\mathbf{k})-\phi_{\mathbf{k}} V\left(\mathbf{q}-\mathbf{k}^{\prime}\right)\right) .
\end{aligned}
$$

We minimize by setting $\partial \phi_{\mathbf{k}}^{*}\langle(H-E)\rangle=0$ and $\partial \kappa_{\mathbf{k k}^{\prime} \mathbf{q}}^{*}\langle(H-E)\rangle=0$ and we get the following coupled equations:

$$
\left(-E+\varepsilon_{F \mathbf{k}}+\varepsilon_{\mathbf{k}}^{I}+V(\mathbf{0}) n_{F}-\epsilon_{F}\right) \phi_{\mathbf{k}}+\sum_{\mathbf{k}^{\prime}}^{\prime} V\left(\mathbf{k}^{\prime}-\mathbf{k}\right) \phi_{\mathbf{k}^{\prime}}+\sum_{\mathbf{k}^{\prime} \mathbf{q}}^{\prime}\left(\kappa_{\mathbf{k k}^{\prime} \mathbf{q}}-\kappa_{\mathbf{k}^{\prime} \mathbf{k q}}\right) V\left(\mathbf{q}-\mathbf{k}^{\prime}\right)=0,
$$

and

$$
\begin{aligned}
-\phi_{\mathbf{k}_{1}} V\left(\mathbf{q}-\mathbf{k}_{\mathbf{2}}\right)+\phi_{\mathbf{k}_{\mathbf{2}}} V\left(\mathbf{q}-\mathbf{k}_{\mathbf{1}}\right)+E_{\mathbf{k}_{1} \mathbf{k}_{\mathbf{2}} \mathbf{q}}\left(\kappa_{\mathbf{k}_{1} \mathbf{k}_{\mathbf{2}} \mathbf{q}}-\kappa_{\mathbf{k}_{\mathbf{2}} \mathbf{k}_{\mathbf{1}} \mathbf{q}}\right)+\sum_{\mathbf{q}^{\prime}}^{\prime}\left(\kappa_{\mathbf{k}_{1} \mathbf{k}_{\mathbf{2}} \mathbf{q}^{\prime}}-\kappa_{\mathbf{k}_{2} \mathbf{k}_{1} \mathbf{q}^{\prime}}\right) V\left(\mathbf{q}^{\prime}-\mathbf{q}\right) \\
+\sum_{\mathbf{k}}^{\prime}\left(\kappa_{\mathbf{k}_{1} \mathbf{k} \mathbf{q}}-\kappa_{\mathbf{k k}_{\mathbf{1}} \mathbf{q}}\right) V\left(\mathbf{k}_{\mathbf{2}}-\mathbf{k}\right)+\sum_{\mathbf{k}}^{\prime}\left(\kappa_{\mathbf{k}_{2} \mathbf{q}}-\kappa_{\mathbf{k}_{\mathbf{2}} \mathbf{k} \mathbf{q}}\right) V\left(\mathbf{k}_{\mathbf{1}}-\mathbf{k}\right)=0,
\end{aligned}
$$

where $E_{\mathbf{k}_{1} \mathbf{k}_{\mathbf{2}} \mathbf{q}}=-E+\varepsilon_{F \mathbf{k}_{1}}+\varepsilon_{F \mathbf{k}_{2}} \varepsilon_{F \mathbf{q}}+\varepsilon_{\mathbf{q}-\mathbf{k}_{1}-\mathbf{k}_{2}}^{I}+V(\mathbf{0}) n_{F}-\epsilon_{F}$. The inclusion of the $\epsilon_{F}$ is because we still choose to the measure the energy $E$ in terms of the $N$ particle energy of the fermi sea, even though there are only $N-1$ particles in the fermi sea for the case of the dressed dimer. 